\title{
Application of Big Data Analysis in Medical and Health Institutions in Chengdu
}

\author{
Yundong Hao \\ School of Economics and Management, Zhejiang Ocean University, Zhoushan, Zhejiang, 316022
}

\begin{abstract}
With the development of social economy, information technology is developing constantly, and the whole society has entered the era of big data. The era of big data is mainly manifested in the large amount of data, various types, low value density, and high requirements for data processing speed and timeliness. In the era of big data, we should extract data in time and effectively, so as to promote the development of various industries. As an institutional industry that plays an important role in the cure of human health diseases, medical and health institutions have begun to gradually use big data to play an important role in the management of people's diagnosis and treatment. Taking medical and health institutions in Chengdu as an example, this paper explores how to use big data analysis in medical and health institutions, so as to improve the efficiency of medical treatment and medical quality.
\end{abstract}

Keywords: Big data, Analysis, Medical institution.

\section{Big Data Related Concepts}

\subsection{Big Data Concept}

Big data is not a simple new technology, but a technological innovation after cloud computing and Internet of Things. The scope of big data includes data, thinking and technology. From the overall point of view, big data is to strengthen the collection of massive data, which requires the implementation of data scale diversity. In the process of emphasizing data scale and diversity, At the same time, it is also necessary to consider the speed and value of data generation. Generally speaking, diversity of scale, growth rate and value are the characteristics of big data. From the perspective of thinking, big data provides a new way to look at the world in the process of our work and life. It is not that samples replace the whole and no longer pursue sample surveys, but grasp the whole. Reduce the accuracy of the individual quantity, so as to know the actual situation of the whole data more accurately. Technically, big data is a technological innovation in the new era. Integration, storage and mining of big data are very innovative technologies, which solve the difficulties and problems that traditional data processing cannot solve. The development and maturity of data accelerate the coming of big data era.

\subsection{Medical Big Data}

At present, the definition of medical big data is not uniform, and different scholars have different definitions of medical big data. Yu Guopei believes that medical big data should be divided into six categories: hospital medical big data, regional health service platform health big data, disease monitoring big data, self-quantified big data, big data network and biological big data. Yan Yan believes, Medical big data refers to the application of data generated by medical personnel in the process of patients' diagnosis and treatment, including behavioral data, diagnosis and treatment data, management data, examination data and electronic medical records, etc. At present, there are some misunderstandings in health care big data. Many people think that clinical data and health data are equivalent concepts. Secondly, some people ignore the application of non-clinical data in the process of application.
Medical big data, by collecting and analyzing specific data of individuals and groups, can effectively promote the development of medical undertakings, and at the same time, it can avoid detours in medical undertakings and promote the efficiency of medical undertakings.

\section{The Current Situation of Big Data Application in Medical and Health Institutions in Chengdu}

At present, the basic database of population in Chengdu is constantly developing and has made new progress. The establishment of population basic database is the core of big data and the foundation of big data. Chengdu has built a full population database to cover the whole population, and besides the establishment of regional big data, Medical institutions in Chengdu are also connected with national information medical big data, and qualified medical institutions can borrow and check personal information at any time. At the same time, Chengdu has also established residents' health records and electronic medical records, and the coverage rate in the city has basically reached $100 \%$. At the same time, Realize the sharing of electronic medical records and the use of electronic health medical records, and strictly record personal medical records and diseases. These two data are mainly about personal diagnosis and treatment, physical condition, related data records, etc., and are made into electronic medical records, which are convenient for timely access and use. At the same time, if the patient has a change in medical institution and condition, you can also access the medical big data through one-click Internet access in time to avoid spending too much manpower and material resources in the process of diagnosis and treatment.

Medical big data is constantly being established among different districts in Chengdu, and the establishment of residents' electronic archives in each district and county has reached $70 \%$ at present. High-tech Zone, Jinniu district, Tianfu New District, Chenghua district, Wuhou District, Qingyang District and Jinjiang District are currently establishing databases, and Tianfu New District and High-tech Zone have been established. Other districts are still under construction. At the same time, the High-tech 
Zone, Tianfu New District and the traditional Wucheng District have realized the exchange and sharing of residents' electronic medical records in the districts, and the residents' health records database and electronic medical records database have been built, which can be accessed and used each other at any time.

Moreover, with the rise of health care and mobile medical care, new wearable or used residents' health data such as sports bracelets, sleeping pillows and sleeping mattresses are increasing, and various forms of disease medical databases are also emerging. In the whole process, medical dataization accelerated the scale development, and Chengdu passed the resident health card, carry out telemedicine and other information benefiting people projects. In-depth promotion of Appointment registration, health clinic, diagnosis inquiry report, physical examination inquiry and health management app, all of which can be used in Chengdu, greatly saving the speed of medical treatment and improving the work efficiency.

\section{The Characteristics of Big Data Application in the Medical Industry}

On the whole, regardless of data format, content source and health care, big data has the characteristics of mass, comprehensiveness, privacy redundancy and so on.

\subsection{Mass}

Simply put, medical big data will involve a country or a region, and all people in all hospitals have TB or even PB level data, which is very large. It can also be the data of only one area, several hospitals or a part of healthy people, or even all the medical data of one hospital. But the reality is, every day, the hospital receives a large number of patients, and the medical data of a central hospital can reach tens of TB. Even if the image data is deleted, there is still a large amount of data above hundreds of levels. With the application of big data in medical institutions, the data of various clinical departments can be interconnected in many cases.

\subsection{Privacy}

In the process of adopting health care big data, it is also necessary to protect the privacy of patients, such as the user name, ID card address and sensitive information of patients. At the same time, the medical conditions, health conditions and diseases of patients must also be protected, so medical and health institutions have privacy in the process of using big data.

\subsection{Comprehensiveness}

Health care big data not only needs extensive coverage in individual health information, but also includes mental health, social adaptation, moral quality and so on, which needs to be carried out comprehensively. At the same time, physical health includes the physical functions of various parts, which requires the collection of medical data, reflecting the comprehensive coverage of health care big data.

\subsection{Redundancy}

In the process of recording the same or similar information in health care big data, the common information often needs to be recorded with examination information unrelated to pathological features. Medical big data shows redundancy, that is, not all medical and health data are core data.

\section{Path Analysis of Big Data Application in Medical and Health Care in Chengdu}

At present, medical big data has been widely used in medical and health institutions, and authorized hospitals can share information with medical big data, and can borrow each other from the national medical and health center data network. With the development of social science and technology, the informationization degree of medical and health field in China has been greatly improved. Chengdu, as the center of Southwest China, the degree of informatization in the field of medical and health care has also been significantly improved. At present, more than 100,000 medical and health institutions in Chengdu have established a dynamic database in the form of statistical direct reporting system, which comprehensively covers medical and health care, human resources, health service utilization and health monitoring. At the same time, the basic information of the diagnosis and treatment population, the medical treatment situation, nearly 200 indicators, such as medical services, were covered, and a massive database of medical and health services was established for the number of candidates and patients in the world. At present, the data processing in the field of health care is mainly carried out in the following aspects.

\subsection{Medical R\&D}

In the application process of big data in the medical and health field, it can carry out specialized processing and targeted analysis, combine with the statistical analysis of a large number of data, and analyze the details of patients' behaviors and emotions, which can improve and deeply grasp personal privacy and disease characteristics, and provide power for making accurate judgments in the later diagnosis and treatment process. It provides powerful data support for patients and doctors to provide more matching drugs in the diagnosis and treatment process, and can optimize and develop the services and research in the medical and health field as a whole. Based on the advantages of big data, Chengdu Medical R\&D Department has tried to use big data in the new drug research stage. For example, Chengdu pharmaceutical R\&D department selectively uses big data technology to support the Internet, judges the demand trend of social drugs, and determines the most scientific input-output ratio in combination with its own $R \& D$ actual situation, so that $R \& D$ costs can be effectively utilized and resources can be optimally allocated. At the same time, Medical R\&D departments use big data technology to manage logistics information, so that capital efficiency can be effectively improved, similar diseases and symptoms can be deeply mined, and valuable data can be provided for later optimization of drug composition ratio. At present, the period from research and development to market entry of traditional new drugs in Chengdu is thirteen years. 
After the application of big data, the time period is obviously reduced, and in an ideal state, the cycle time can be effectively shortened by about $20 \%$.

\subsection{Adopt Information Management Mode}

At present, the use of big data is gradually deepening, which also improves the information management mode of medical and health institutions in the medical network. On the basis of learning and introducing foreign advanced management ideas, medical and health institutions in Chengdu carry out medical quality management according to the actual situation. For example, through real-time control of medical quality in West China Hospital, collect real-time data of medical quality and conduct medical management. Traditional medical quality control focuses on terminal quality evaluation and feedback, and seldom involves link quality control. Because quality control cannot be reflected from data, management efficiency is relatively low. The real-time control of medical quality is based on the basic theory of cybernetics and information theory, by combining hospital medical quality management with computer technology, a new medical quality management mode is established, that is, a real-time medical quality control system which integrates feedforward control, feedback control and field control of medical process. To realize the effective monitoring and control of the real-time information of medical quality by the hospital decision-making, management and executive levels. It overcomes the shortcoming that traditional quality management only pays attention to "governance", and can establish a quality control system that can both "governance" and "prevention". By strengthening process management, it can play the role of "prevention", in order to achieve the purpose of improving the medical quality level more effectively.

\subsection{Public Health Management}

It is an important responsibility of public health management to grasp the data accurately and timely, so as to forecast and warn. By collecting massive data, big data can effectively integrate and analyze data, apply it to the public health field, and improve the quality of task completion. Chengdu public health department has applied big data to the comprehensive health management platform. It can also be used in residents' health database to process the basic information of patients' illness and medical records, which can effectively improve the speed of infectious disease monitoring and improve the comprehensiveness of epidemic monitoring. In the process of use, the probability of medical claims and residents' disease infection is obviously reduced, which promotes the development of public health management. At the same time, combining with the results of big data, developing public-oriented health consultation services in Chengdu can effectively enhance public risk awareness and lay a mass foundation for improving the efficiency of public health management.

\subsection{Establish Resident Health Records}

Big data has effectively promoted the development of individuation. The major health and medical institutions use big data to establish residents' health records, which is led by the medical departments of Chengdu government, so that the data health of residents in physical examination can be reflected in the form of big data, saving time in the process of diagnosis and treatment, and making modern nutrition widely recognized. In the process of building big data health records, the public also recognizes the impact of psychological environment and nutrition exercise on health, and can combine big data processing information with their own actual situation to enhance their health awareness. At the same time, big data can effectively integrate patient information, conduct remote diagnosis, and optimize treatment services. Combined with mobile devices, the positioning data are processed, and the causes of diseases are comprehensively analyzed, which makes online residents' health management possible.

\section{Problems and Challenges in the Application of Big Data in Medical and Health Institutions-Taking Chengdu as an Example}

By analyzing the application status of medical big data in Chengdu medical and health institutions, it is not difficult to find that the application of big data in Chengdu medical and health institutions has become more popular, but there are still great differences in the application depth and intensity of big data database. Some top three hospitals, such as West China Hospital and Provincial Hospital of Traditional Chinese Medicine, are relatively more stable and radical with the introduction of big data and the establishment of the platform at home. However, for some private hospitals and small medical clinics, due to lack of manpower, material resources and financial resources, they are still unable to introduce big data. In the era of big data, It is necessary to change the data processing methods used in the traditional database era. From database to big data, it is not only the technological evolution, but also the essential differences. The emergence of big data will bring subversive changes to traditional data management methods, and bring revolutionary changes in data sources, processing methods and thinking. This change also brings more severe challenges to the application of big data technology in medical institutions and medical and health fields in the future.

\subsection{Technical Operation Problems}

First of all, for data, big data must reduce errors in the integration process and ensure the quality. Errors are not allowed in the era of big data, which has become a new bright spot. Under the medical and health sharing platform, many medical and health institutions will have a lot of data, and it is very difficult to collect and integrate them. The data quality of the existing big data platform is not ideal. For everyone, the historical diagnosis and treatment data of personal information must be accurate. If mistakes occur, it is very likely that the quality of medical care will decline. At present, the guarantee of correctness and authenticity in the process of data acquisition, it is a thorny problem and 
challenge for the application of big data technology in medical and health institutions.

At the same time, from the perspective of Chengdu, the expanding medical data is mixed with a large number of unstructured data, and the data sources are more and more diverse. The storage architecture can no longer meet the needs of big data applications, and in many cases, the query process is inadequate. For example, the capacity problem is very obvious, and the mass data storage system should have the corresponding level expansion capability. In addition to the huge data scale, it also needs to have a huge number of files, so how to manage it is also a difficult problem. Medical big data needs to pay attention to real-time problems in the application process, and it needs to be processed in real time and queried in seconds, which requires very high data and technology. Meanwhile, medical big data itself is unstructured data, Traditional structured database can't meet the storage requirements, so hospitals and medical institutions need to re-integrate the database system and mine data. Current regional health information platform data mainly include direct utilization and indirect utilization. Direct utilization requires information access and sharing to assist medical services. At the same time, collaborative services based on information sharing services are needed. Indirect utilization mainly analyzes the performance according to the needs of medical and health administration. Effective data mining for unstructured data is also a difficulty and a challenge in implementation.

\subsection{Users Lack Scientific Knowledge of Big Data}

The application of medical data technology often requires people's participation, such as stimulation and supervision. Although medical and health institutions have begun to use big data, many doctors and staff do not have enough awareness to use big data, at the same time, they do not have enough awareness of the significance of these data and do not accurately grasp the operational characteristics of big data technology. The government itself has begun to adopt stimulating payment and compensation plans to encourage everyone to carry out electronic health records, but there are always some problems in the process of transformation, which is also an obstacle encountered in the implementation process. Due to the lack of awareness of big data among users, there are some problems such as improper operation.

\subsection{Information Cannot be Connected}

China has a large population, and the medical and health field is a huge and complex field. There are a wide range of big data users in the medical field, such as hospitals, clinics, medical centers, insurance companies, drug management, etc., all of which are the main applications, and the corresponding data resources are scattered in different data fields. Including medical record settlement data, expense data, manufacturer data, mechanical data, residents' health records, population and health data investigated by the government, etc., the relationship between them has not been effectively established, and the information is still isolated.

\section{The Strategy of Improving the Application Effect of Big Data Analysis in Medical and Health Field}

\subsection{Improve the Network Security Organization and Management System}

Strengthen the construction of industry information security talents. The unit shall set up special network management institutions and posts, define the key posts of network security, the distribution of responsibilities, the requirements of employee skills and the number of employees, and train industry security experts. Establish an industry network security special control team, and regularly or irregularly carry out the security evaluation and risk assessment of important information systems in the industry.

\subsection{Defend the Information System in Depth and Strengthen the Privacy Protection of Medical Information Data}

For the transmission and storage of information, effective network isolation measures are deployed, different access users of the system are strictly classified in terms of authority, data protection capabilities in safety awareness, safety disposal and safety audit are improved, and supervision of third-party safety operation and maintenance units is strengthened. Therefore, it can optimize and analyze the problems of privacy protection in the usage of big data in medical industry. At the same time, medical institutions should be defined as health care big data managers and operators in the legal sense. This will be beneficial to the privacy protection of personal health data and the free access of the owner, and facilitate the second-party diagnosis and participation in the health cloud plan. By authorizing the circulation of basic data, breaking the barriers of big data islanding effect is conducive to urging medical institutions to actively promote the informationization process, actively establish complete electronic files and electronic medical records, and lay a legal basis and effective assets for the application and circulation of big data.

\subsection{Do a Good Job in the Classification of Medical and Health Big Data to Improve the Efficiency of Use}

Medical health big data can be divided into: hospital medical big data: including various outpatient and emergency records, hospitalization records, image records, laboratory records, medication records, surgical records, follow-up records and medical insurance data; Regional service platform medical health big data; Disease surveillance big data: Big data based on medical research or disease monitoring of a large number of people; Self-quantified big data: Self-quantified data based on personal physical signs and activities of the mobile Internet of Things, including blood pressure, heartbeat, blood sugar, breathing, sleep, physical exercise and other information; Internet and social media data: refers to various medical-related data on the Internet; Biological big data: It is mainly about biological specimens and gene sequencing information, which is directly related to clinical personalized 
diagnosis and treatment and precision medicine; Insurance data: medical insurance, new rural cooperative medical system, health insurance data, etc. External data: including environmental, meteorological and geographical data, etc. In the process of using medical big data in the future, it is necessary to continuously improve its classification research and data fusion. Thereby promoting the joint use of medical big data.

With the development of social economy, big data technology is also improving. At present, various industries are constantly improving the efficiency and intensity of data use, which provides convenience for the fine development of various industries. Taking medical institutions in Chengdu as an example, this paper explores the application status and characteristics of big data analysis in medical institutions in Chengdu. Explore the specific problems in the application process, put forward constructive suggestions, promote big data analysis technology to better serve the development of medical industry, constantly overcome the difficulties in technology, management and use, and promote the continuous progress and development of China's medical industry and service level.

\section{References}

[1] Liang Yimin. Application and challenge of medical big data $[\mathrm{J}]$. Electronic Technology and Software Engineering, 2018 (06).

[2] Jin Chunlin, Peng Ying. Online+Offline: medical services are more accurate and convenient $[\mathrm{J}]$. Chinese Hygiene, 2018 (06).

[3] Ma Guoyao, Sun Yongtao, Ma Yuling. Analysis of the application of data verification technology in the quality control of medical and health big data $[\mathrm{J}]$. Chinese Journal of Health Information Management, 2016 (04).

[4] Li Zhirong. Integrated "Smart Medical Health Qingdao" mode [J]. Software and Integrated Circuit, 2017(05)

[5] Gao Yanjie, Shen Jing. Analysis of the development of medical and health websites [J]. Chinese Hospital Statistics, 2001 (02).

[6] Li Yazi, Tian Binglei, Li Yanling, Xia Jinghui, Qian Qing. Research on security and privacy protection in secondary utilization of medical health information [J]. Journal of Medical Informatics, 2014 (09).

[7] Li Ziyang. Analysis of the current situation and trend of medical health development in 2017-2018 [J]. Smart Health, 2018 (15).

[8] Li Huacai. Discussion on improving the understanding of the true value of medical and health big data [J]. China Digital Medicine, 2018 (08).

[9] Li Huacai. A brief talk on the construction of medical and health big data ecosystem [J]. China Digital Medicine, 2017 (04).

[10] Du Mingchao, Hong Jian, Yan Yuchun, Zhou Dian, Yin Dong, Tao Min. Application scope and value analysis of health care big data [J]. Chinese Journal of Health Information Management, 2017 (05).

[11] Long Hu, Qiu Hang, Wu Canglang, Hu Juan, Tank Tan, Deng Ren. Discussion on the construction of big data center for health care in Sichuan Province [J]. Chinese Journal of Health Information Management, 2017 (01).

[12] Meng Qun, Bi Dan, Zhang Yiming, Yin Xin. Research on the development status and application mode of health care big data $[\mathrm{J}]$. Chinese Journal of Health Information Management, 2016 (06)

[13] Zhang Guoming, Chen Anqi. Exploration of medical big data utilization based on regional health information platform [J]. Chinese Journal of Health Information Management, 2016 (03).

[14] Liao Yufeng. Analysis of data governance model in big data environment $[\mathrm{J}]$. Journal of Library and Information Sciences in Agriculture, 2016 (06).

[15] Zhang Mingying, Pan Rong. Interpretation of key points of international standards research report of white paper on data governance [J]. Information Technology and Standardization, 2015 (06).

[16] Kong Bin, Cai Jiahui, Zong Wenhong. Practice and thinking of data quality control in regional health information platform [J]. Chinese Journal of Health Information Management, 2014 (02).

[17] Liu Xiaoliang, Kun Wang, Ma Jun. Thinking on health information construction in the era of big data $[\mathrm{J}]$. Chinese Journal of Health Information Management, $2014(01)$

[18] Li Guojie, Cheng Xueqi. Big data research: a major strategic field of future science and technology and economic and social development-research status and scientific thinking of big data $[\mathrm{J}]$. Proceedings of Chinese Academy of Sciences, 2012 (06).

[19] Zhu Lingyun, Wu Baoming. Technology, method and application of medical data mining [J]. Journal of Biomedical Engineering, 2003(03).

\section{Author Profile}

Yundong Hao (1997-), Graduate student of School of Economics and Management, Zhejiang Ocean University. Xuchang, postgraduate, research direction: rural social development. 